\title{
GYROSCOPICS AS A MEANS OF DEMONSTRATING ROTATIONAL INSTABILITY IN STARS
}

\author{
M.S.A. SASTROAMIDJOJO \\ Solar Physics Laboratory, Gadjah Mada University \\ Yogyakarta, Central Java, Indonesia
}

\begin{abstract}
Light-traces of a light bulb fixed on top of the stem of a spinning top were recorded to simulate rotation. It was found that with dissipation of energy the rotational frequency did not decrease monotonically, but showed 'quasi-periodic' sequences.
\end{abstract}

\section{Introduction}

A star is a physical entity of gaseous material hold together by the force of gravity, more or less spherical in shape. A spinning top given initial rotational energy and left by itself will display such features as spin, rotation and precession. It was with this in mind that we tried to simulate stellar rotation in the laboratory, because not only could basic mechanical dynamics be demonstrated, but also phenomena far from equilibrium.

\section{Method}

The basic set-up needed the following paraphernalia: (1) a gyroscope made of a bicycle wheel with a light bulb on the top of the stem and two flashlight batteries in the centre of mass; (2) a square grid of chicken wire with a $2 \times 2 \mathrm{~cm}$ mesh, providing a frame of reference; (3) a 35- $m m$ Pentax camera; (4) a ground plate with cup for sustaining the lower end of the gyroscope stem; (5) a distance of $70 \mathrm{~cm}$ from centre of mass to top of stem and a distance of $20 \mathrm{~cm}$ from centre of mass to lower end.

The procedure was as follows: (1) the light bulb was switched on; (2) the gyroscope was set spinning; and (3) the light-trace was recorded through the wire mesh with 3 -second exposures at 5 -second intervals, till the gyroscope stopped rotating.

\section{Results}

The recorded light-traces show a quasi-periodic start with a concentration of light in the center, followed by alternating 'widening' and 'bunching' of the precessions. The same can be said about the rotational frequency, which went through an 'up-and-down' sequence, according to the movement of the gyroscope stem, from equilibrium (standing almost vertical) to far from equilibrium (almost falling down).

It is suggested that the 'new' discipline of 'synergetics', chaos-order-chaos, may perhaps be studied with the gyroscope model.

\section{Acknowledgement}

The experiments were done by Indonesian Islamic University (UII, Yogyakarta) first year engineering students, using the facilities of the Gadjah Mada Solar Physics Laboratory. 